\title{
Drug Safety of Celecoxib
}

\author{
Bindu K.H' ${ }^{1}$, Rao G. $\mathbf{V}^{2}$ \\ ${ }^{1}$ Dr. K. Hima Bindu, Associate Professor, Department of Pharmacology, affiliated with Kakatiya Medical College, \\ Warangal, Telangana State, India ${ }^{2}$ Dr. G. Venkat Rao, Orthopedics, Retired Associate Professor in Orthopedics, House \\ No.2-10-790/10, Teachers' Colony, Phase 1, Subedari, Hanumakonda, Warangal - 506007, Telangana State, India
}

Address for Correspondence: Dr. K. Hima Bindu, Associate Professor, Department of Pharmacology, Kakatiya Medical College, Warangal, Telangana State, India. Email address: hk.himabindu@gmail.com

\begin{abstract}
Introduction: Non-steroidal anti-inflammatory drugs presently are the most widely used drugs in medicine and are the most frequent cause of adverse drug reactions, affecting multiple systems, mainly gastric, renal \& cardiovascular, which is a major public health concern. Previous studies report, that selective COX-2 inhibitors are safer when compared to non-selective cyclooxygenase inhibitors. But, recent studies reveal, that the safety of these selective COX-2 inhibitors is not much better than that of conventional NSAIDs. In view of the wider usage of selective COX-2 inhibitor, celecoxib in day to day practice by many clinicians, the study has been taken up to report, whether selective COX-2 inhibitor celecoxib has got any advantages over conventional NSAIDs or not. Methodology: 15 patients were taken up for the study \& all the patients were given celecoxib for 15 days. Both pre and post-treatment values of $\mathrm{Hb} \%$, blood urea, serum creatinine, serum bilirubin, bleeding time, clotting time, heart rate \& blood pressure were recorded, tabulated \& subjected to statistical analysis. Results: Selective COX-2 inhibitor, celecoxib has shown significant changes in bleeding time values \& also good amount of gastric toxicity when assessed clinically. Hepatic, renal \& other cardiovascular parameters were absolutely normal. Conclusion: In our short-term study, selective COX-2 inhibitor, celecoxib has shown good amount of gastric toxicity, showing no advantage over non-selective drugs. But, there is no hepatic, renal \& cardiovascular toxicity.
\end{abstract}

Keywords: Adverse drug reactions, COX-2 inhibitors, Cardiovascular Toxicity, Celecoxib, Gastric toxicity

\section{Introduction}

Non-steroidal anti- inflammatory drugs presently are the most widely used drugs in medicine. Some of which are often taken without prescription for simple conditions like even minor aches \& musculoskeletal pains, as over the counter drugs. Nearly 35 million people are taking them on daily basis and FDA has ranked them the most frequent cause of adverse drug reactions [1]. Celecoxib, one of the selective COX-2 inhibitors is approved for the treatment of osteoarthritis and rheumatoid arthritis.

The recommended dose for treating osteoarthritis is 200 $\mathrm{mg}$ per day as a single dose or as two $100 \mathrm{mg}$ doses. In the treatment of rheumatoid arthritis, the recommended dose is 100 to $200 \mathrm{mg}$ twice per day [2].

Manuscript received $10^{\text {th }}$ September 2016

Reviewed: $20^{\text {th }}$ September 2016

Author Corrected: $4^{\text {th }}$ October 2016

Accepted for Publication $17^{\text {th }}$ October 2016
Effect on Gastrointestinal system: Adverse gastrointestinal events are the commonest unwanted effects of the NSAIDs, and are believed to result mainly from the inhibition of gastric COX-1, which is responsible for the synthesis of prostaglandins that normally inhibit acid secretion and protect the mucosa.

These commonly include gastric discomfort, dyspepsia, diarrhea (but sometimes constipation), nausea and vomiting, and in some cases gastric bleeding and ulceration. It has been estimated that $34-46 \%$ of users of NSAIDs will sustain some gastrointestinal damage that, while it may be asymptomatic, carries a risk of serious hemorrhage or perforation (Fries, 1983). Severe gastrointestinal effects (perforations, ulcers or bleeding) are said to result in the hospitalization of over 100000 people per year in the USA [3]. All selective COX-2 inhibitors are less prone to induce endoscopically 
visualized gastric ulcers than equally efficacious doses of conventional NSAIDs (Deeks et al., 2002) and this has provided the basis of FDA approval of selective COX-2 inhibitor, celecoxib [2].

CLASS and VIGOR trials showed a lower relative risk of serious gastro intestinal complications with selective COX-2 inhibitors [4, 5, 6, 7 and 8]. Furthermore, subsequent analysis of CLASS study, highlighted by FDA found that the benefits shown in six months analysis were not continued to the twelve months end point of study, placing doubt on the clinical significance of any gastro intestinal safety benefits from chronic use of selective COX-2 inhibitors over traditional NSAIDs [9].

Effect on Renal system: NSAIDs are associated with loss of the prostaglandin-induced inhibition of both the reabsorption of $\mathrm{Cl}$ - and the action of anti-diuretic hormone, leading to the retention of salt and water. Experiments in mice that attribute the generation of vasodilator prostaglandins $\left(\mathrm{PGE}_{2}\right.$ and $\left.\mathrm{PGI}_{2}\right)$ to $\mathrm{COX}-2$ raise the likelihood that the incidence of hypertensive complications (either new onset or worsened control) induced by NSAIDs in patients may correlate with the degree of inhibition of COX-2 in the kidney and the selectivity with which it is attained (Qi et al., 2002). Deletion of receptors for both $\mathrm{PGI}_{2}$ and $\mathrm{PGE}_{2}$ elevate blood pressure in mice, mechanistically integrating hypertension with a predisposition to thrombosis [10].

Conventional NSAIDs and COX-2 inhibitors have been associated with renal and renovascular adverse events (Cheng and Harris, 2004).Therapeutic doses of NSAIDs in healthy individuals pose little threat to kidney function, but in susceptible patients with congestive heart failure, hepatic cirrhosis, chronic kidney disease, hypovolemia, and other states of activation of the sympathoadrenal or renin-angiotensin systems, prostaglandin formation becomes crucial in model systems and in humans (Patrono and Dunn, 1987) \& thus they cause acute renal insufficiency, which is reversible on discontinuing the drug.

This occurs through the inhibition of the biosynthesis of those prostanoids $\left(\mathrm{PGE}_{2}\right.$ and $\mathrm{PGI}_{2} ;$ prostacyclin) involved in the maintenance of renal blood flow, specifically in the $\mathrm{PGE}_{2}$-mediated compensatory vasodilatation that occurs in response to the action of noradrenaline (norepinephrine) or angiotensin II. [3, 2]. Although this hypothesis has never been addressed directly, epidemiological studies suggest that, hypertensive complications occur more commonly in patients treated with coxibs than with conventional NSAIDs. The hypertension is secondary to inhibition of COX-2 in the renin-secreting macula densa region of the kidney \& is dose and time-dependent. [2]

Effect on Cardiovascular system: Placebo-controlled trials have established that celecoxib confers a risk of myocardial infarction and stroke and this appears to relate to dose and the underlying risk of cardiovascular disease that anteceded drug administration. The risk of thrombosis, hypertension, and accelerated atherogenesis are mechanistically integrated. The coxibs should be avoided in patients prone to cardiovascular or cerebrovascular disease. None of the coxibs has established superior efficacy over conventional NSAIDs. Celecoxib carries the same cardiovascular and gastrointestinal black box warning as conventional NSAIDs [10].

Selective inhibitors of COX-2 depress $\mathrm{PGI}_{2}$ formation by endothelial cells without concomitant inhibition of platelet thromboxane. Experiments in mice suggest that $\mathrm{PGI}_{2}$ restrains the cardiovascular effects of $\mathrm{TXA}_{2}$, affording a mechanism by which selective inhibitors might increase the risk of thrombosis.

This mechanism should pertain to individuals otherwise at risk of thrombosis, such as those with rheumatoid arthritis, as the relative risk of myocardial infarction is increased in these patients compared to patients with osteoarthritis or no arthritis. The incidence of myocardial infarction and stroke has diverged in such at-risk patients when COX-2 inhibitors are compared with conventional NSAIDs (FitzGerald, 2003). Placebocontrolled trials have now revealed an increased incidence of myocardial infarction and stroke in patients treated with celecoxib (Solomon et al., 2005) consistent with a mechanism-based cardiovascular hazard for the class (FitzGerald, 2003).

Regulatory agencies in the United States, Europe, and Australia have reviewed these studies and other available evidence and have concluded that selective COX-2 inhibitors increase the risk of heart attack and stroke and will be labeled accordingly and restricted with respect to marketing directly to consumers. Patients at increased risk of cardiovascular disease or thrombosis are particularly prone to cardiovascular adverse events on these agents. [2] 
The cardiovascular hazard from celecoxib, a selective COX-2 inhibitor for which data is available from placebo-controlled trials lasting more than 1 year increased with chronicity of dosing. This is consistent with a mechanism-based acceleration of atherogenesis directly via inhibition of $\mathrm{PGI}_{2}$ and indirectly due to the rise in blood pressure consequent to inhibition of COX2 derived $\mathrm{PGE}_{2}$ and $\mathrm{PGI}_{2}$.

If COX-2 inhibitors are selected, they should be used at the lowest possible dose for the shortest period of time, and patients at risk of cardiovascular disease or prone to thrombosis should not be treated with these drugs. [2] Previous studies report, that selective COX-2 inhibitors are safer when compared to non-selective cyclooxygenase inhibitors.

But, recent studies reveal, that the safety of these selective COX-2 inhibitors is not much better than that of conventional NSAIDs. In view of the wider usage of selective COX-2 inhibitor, celecoxib in day to day practice by many clinicians, the study has been taken up to report, whether selective COX-2 inhibitor celecoxib has got any advantages over conventional NSAIDs or not.

\section{Aims and Objectives}

To know, whether selective COX-2 inhibitor celecoxib has a greater safety profile or not, regarding its effect on gastrointestinal, renal \& cardiovascular systems.

\section{Materials and Methods}

An interventional \& prospective study was taken up with the approval of institutional ethical committee. All patients included, in our study gave informed consent for their participation and the study was done at Mahatma Gandhi Memorial Hospital, Warangal.

\section{Subjects selected for the study:}

\section{Inclusion criteria}

- Patients of above 30 years age; of either sex

- Osteoarthritis patients
- Rheumatoid arthritis patients

- Patients with fractures and dislocations

\section{Exclusion criteria}

- History of acid peptic disease

- History of bronchial asthma

- History of bleeding disorders

\section{Drug used in the study and it's dose:}

\section{CELECOXIB $100 \mathrm{mg}$ bd (200mg per day)}

All the 15 patients were given celecoxib $100 \mathrm{mg}$ bd (200mg / day) \& the following tests were done in all the patients, prior to the administration of drug.

- Hemoglobin percentage- by Cyan methhemoglobin method

- Blood Urea-by Phosphotungstate method

- Serum Creatinine- by Alkaline picrate method

- Serum Bilirubin- by Modified Jen drassik-grof method

- And also Heart rate \& Blood Pressure readings were taken manually both before \& after treatment.

The patients were advised to take celecoxib $100 \mathrm{mg}$ bd, for a period of fifteen days, and they were not put on any antacid or $\mathrm{H}_{2}$ - blocker or proton pump inhibitor. All the out patients were briefed, of the common toxicity of celecoxib and they were advised to inform us soon after the onset of symptoms like nausea, vomiting, diarrhea, epigastric pain, heartburn, dyspepsia, facial and pedal edema, headache and tinnitus.

Whenever the patient reported an adverse effect, it was taken as the primary end point of our study for that particular patient and he or she was advised to stop the treatment.

After the completion of treatment, all the parameters were repeated again and the values were recorded, tabulated and the results were subjected to statistical analysis, with the help of paired 't' test.

\section{Results}

Among all these parameters, only bleeding time has shown significant changes with proper statistical analysis. All the other parameters have shown non-significant changes.

Apart from these, the gastric \& renal toxicity parameters were assessed clinically by taking proper history from the patient \& entering it into a properly designed case sheet proforma. 
Table I: Statistical analysis of Celecoxib data

\begin{tabular}{|l|c|}
\hline Parameters tested & 'p' value \\
\hline 1) $\mathrm{Hb} \%$ & 0.12 \\
\hline 2) Blood Urea & 0.414 \\
\hline 3) Serum Creatinine & 0.58 \\
\hline 4) Serum Bilirubin & 1.0 \\
\hline 5) Bleeding Time & $0.014^{*}$ \\
\hline 6) Clotting Time & 0.149 \\
\hline 7) Heart Rate & 0.103 \\
\hline 8) Systolic Blood Pressure & 0.33 \\
\hline 9) Diastolic Blood Pressure & 0.58 \\
\hline
\end{tabular}

\section{Gastric toxicity parameters:}

- Nausea

- Vomiting

- Abdominal pain

- Heartburn

- Dyspepsia

- Hematemesis

- Constipation

- Diarrhea

\section{Renal toxicity parameters:}

- Edema

- Decreased effectiveness of antihypertensive medications

- Decreased effectiveness of diuretic medications

Table II: Gastric \& Renal toxicity

\begin{tabular}{|l|l|}
\hline Gastric toxicity & 2 \\
\hline Renal toxicity & 0 \\
\hline
\end{tabular}

Selective COX-2 inhibitor celecoxib has shown good amount of gastric toxicity, but no renal toxicity, when assessed clinically.

\section{Discussion}

Effect on Gastrointestinal system: In our study, celecoxib, a selective COX-2 inhibitor showed gastro intestinal toxicity similar to that of non-selective NSAIDs, with no significant change in $\mathrm{Hb} \%$ values [11, 12, Tables I \& II].

Gastrointestinal toxicity by NSAIDs is linked to inhibition of COX-1. Those drugs, which selectively inhibit COX-2, were thought to have less gastro intestinal toxicity. But, they still had sufficient COX-1 inhibition to cause potent inhibitory effects on gastric $\mathrm{PGE}_{2}$ synthesis and thus are responsible for gastric toxicity, similar to that of non-selective NSAIDs. Hence, all currently used NSAIDs do have inherent gastric toxicity, at therapeutic concentrations.

The gastro intestinal toxicity of selective COX-2 inhibitor celecoxib was similar to that of non-selective NSAIDs. [11, 12] FDA also found that, for upper gastro 
intestinal safety, there does not appear to be any meaningful advantage for celecoxib [11]. None of the coxibs has established clinical efficacy over conventional NSAIDs, while celecoxib has failed to establish superiority over conventional NSAIDs in reducing gastrointestinal adverse events.[2]

In our short-term study, celecoxib has proven to have good amount of gastric toxicity, with no hepato toxicity.

Effect on Renal system: NSAIDs are known to be nephrotoxic. Effects attributed to inhibition of PG production in the kidney-hypertension and edemaoccur with nonselective $\mathrm{COX}$ inhibitors and also with celecoxib. But, in our short-term study, celecoxib, a highly selective COX-2 inhibitor neither shown to have any increased risk of salt and water retention nor any significant change in both blood urea \& serum creatinine values $[13,14$, Tables I \& II].

\section{Effect on Cardiovascular system:}

Effect on Bleeding time and Clotting time: Nonselective NSAIDs are associated with risk of prolonged bleeding time. So, in patients who do not require platelet inhibition, selective COX-2 inhibitors seem to be a true advance and an attractive alternative to conventional NSAIDs, combined with other gastroprotective strategies.

But in our short-term study, contrary to it, highly selective COX-2 inhibitor, celecoxib, has shown significant change in bleeding time values, but not with clotting time values; may be due to the fact that there is no significant hepatic effect in our short-term study [Table I].

Effect on Heart Rate and Blood Pressure: There is no significant change in heart rate and blood pressure values in our short-term study, with selective COX-2 inhibitor celecoxib [Table I].

\section{Conclusion}

In our short-term study, celecoxib, a selective COX-2 inhibitor showed gastro intestinal toxicity similar to that of non-selective NSAIDs, with no significant change in $\mathrm{Hb} \%$ values.

No changes in renal function tests have been observed. But, NSAIDs may cause impairment of renal function, in patients suffering from hypovolemia, congestive heart failure or hepatic cirrhosis, since renal function in these patients may be dependent on the vascular effects of prostaglandins.

There is no risk of cardiovascular complications with selective COX-2 inhibitor, celecoxib in our short-term study. Further long-term studies are required to assess the clinical safety of celecoxib.

Funding: Nil, Conflict of interest: None initiated, Permission from IRB: Yes

\section{References}

1. V. Dhikav, S. Singh, KS. Anand; Newer nonsteroidal anti-inflammatory drugs - A review of their therapeutic potential and adverse drug reactions; Journal, Indian Academy of Clinical Medicine; October -December 2002; -Volume 3; No. 4; Page 332-338.

2. Goodman and Gilman; Analgesic-Antipyretic and Anti-inflammatory agents; The Pharmacological Basis of Therapeutics; Eleventh Edition; Page 671715.

3. HP Rang and MM Dale; Anti-inflammatory and immunosuppressant drugs; Rang and Dale's Pharmacology; Seventh Edition: Page 318-335.

4. Jonathan J Deeks, Lesley A Smith, Matthew D Bradley; Efficacy, tolerability, and upper gastrointestinal safety of celecoxib for treatment of (Osteoarthritis and rheumatoid arthritis; systematic review of randomized controlled trials; British Medical Journal; 21 September, 2002; Volume 325; Page 619-623.

5. LLM waeky, JE Ruiz, J Duperly e1.al; Efficacy of celecoxib in treating symptoms of viral pharyngitis; a double-blind, randomized study of celecoxib versus diclofenac; The Journal of International Medical Research; 2002; Volume 30; Page 185-194.

6. Maarten Boers; NSAIDS and selective COX-2 inhibitors: competition between gastro protection and cardio protection; The Lancet; 21 April; Volume 357; Page 1222-1223.

7. Paul Emery, Henning Zeidler, Tore K Kvien, Mario Guslandi, Raphael Naudin, Helen Stead, Kenneth M Verburg, Peter C Isakson, Richard C Hubbard, G Steven Geis; Celecoxib versus diclofenac in long-term 
management of rheumatoid arthritis; randomized double-blind comparison; The Lancet; 18/25 December, 1999; Volume 354; Page 2106-2111.

8. Peter Juni, Anne WS Rutjes, and Paul A Dieppe; Are selective COX-2 inhibitors superior to traditional non-steroidal anti-inflammatory drugs?; British Medical Journal; 1 June, 2002; Volume 324; Page $1287-1288$.

9. Christopher Cutts, Adam Lacaze and Susan Tett; A Clinical audit of the prescribing of celecoxib and rofecoxib in Australian rural general practice; British Journal of Clinical Pharmacology; 2002; Volume 54; Page 522-527.

10. Goodman and Gilman; Analgesic - Antipyretic and Anti-inflammatory agents; The Pharmacological Basis of Therapeutics;Twelth Edition; Page 959-1004.
11. Jennifer Berg Hrachovee, Pharm D, MPH; Marc Mora, MD; Reporting of 6 -months VS 12-months data in a clinical trial of celecoxib; JAMA, 21 November, 2001; Volume286; No.19;Page2398.

12. Mark Feldman, MD, and Alexander T. Mc Mahon, MBA; Do cyclooxygenase inhibitors provide benefits similar to those of traditional non-steroidal antiinflammatory drugs, with less gastro intestinal toxicity?; Annals of Internal Medicine; 18 January 2000; Volume132; No.2; Page 134-143.

13. Harri Vainio, Gareth Morgan; Cyclo-oxygenase- 2 and breast cancer prevention ; British Medical Journal; 26 September, 1998; Volume 317; Page 828.

14. Peter M. Brooks, M.D; And Richard 0.Day,M.D; Non-Steroidal anti-inflammatory drugs-Differences and similarities; the new England Journal of Medicine;13June, 1991;Volume 324; No. 24; Page1 716-1724.

\section{How to cite this article?}

Bindu K.H, Rao G.V. Drug Safety of Celecoxib. Int J Med Res Rev 2016;4(10):1796-1801.doi:10.17511/ijmrr. 2016.i10.15. 